The Annals of Probability 1997, Vol. 25, No. 3, 1257-1283

\title{
STOCHASTIC INTEGRALS: A COMBINATORIAL APPROACH
}

\author{
By Gian-Carlo Rota AND Timothy C. Wallstrom \\ Massachusetts Institute of Technology, Los Alamos National Laboratory and \\ Catholic University of America and Los Alamos National Laboratory
}

\author{
Dedicated to W. T. Martin
}

\begin{abstract}
A combinatorial definition of multiple stochastic integrals is given in the setting of random measures. It is shown that some properties of such stochastic integrals, formerly known to hold in special cases, are instances of combinatorial identities on the lattice of partitions of a set. The notion of stochastic sequences of binomial type is introduced as a generalization of special polynomial sequences occuring in stochastic integration, such as Hermite, Poisson-Charlier and Kravchuk polynomials. It is shown that identities for such polynomial sets have a common origin.
\end{abstract}

1. Introduction. Few subjects in modern probability have undergone as many disparate presentations and have been rediscovered in as many different guises as the theory of stochastic integrals. Wiener's homogeneous chaos [38], Wiener and Wintner's discrete chaos [39], the Fock spaces of quantum field theory [2], Itô's stochastic integrals [13, 14], integration over semimartingales [24, 28, 7, 4], Segal's tensor algebras over Hilbert spaces [34], Kakutani's maximal Gaussian subspaces [15, 16], are only some of the theories that have evolved in the last fifty years around one fundamental idea [23, 20, 21, 22, 25, $12,18]$.

The variety of notations, ranging from Cameron and Martin's products of Hermite polynomials [3] to Wick's "dots" [37], has obscured the basic simplicity of the underlying concept. What is more, the lack of communication among various schools, notably between physicists aiming at the development of nonlinear quantum field theories $[36,40]$ and probabilists in search of new point processes that would not turn out to be Poisson distributions in disguise $[17,6]$, have delayed and in some cases prevented a full understanding of the possibilities of stochastic integration.

The purpose of the present work is modest. It is to present the basic idea of stochastic integration in what we believe to be its simplest and most natural form, stripped of all analytic superstructure and of all physical irrelevancies. Not surprisingly, we have found that the basic infrastructure of stochastic integration is a combinatorial technique which had heretofore remained hidden, namely, Möbius inversion over the lattice of partitions of a set [30]. Insofar as the underlying analytic machinery goes, we rely heavily on the pioneering

Received March 1996.

AMS 1991 subject classifications. Primary 60H05, 05A18; secondary 05E 05, 05E 35, 11B65, 60G57, 81T18.

Key words and phrases. Multiple stochastic integrals, partitions of sets, discrete and homogeneous chaos, orthogonal polynomials, symmetric functions, Kailath-Segall formula. 
work of Engel [9], whose proof of countable additivity of product measures allows us to proceed directly to the combinatorial and algorithmic aspects of stochastic integration.

Systematic use of Möbius inversion not only simplifies definition of multiple stochastic integrals and their computation, but displays the unity of the ad hoc formulas that seemed to vary capriciously from one example to the next. In the presentation given below, identities holding among the special functions that had arisen in various contexts of stochastic integration are shown to stem from one and the same set theoretic identity. Thus, Hermite, PoissonCharlier, Kravchuk and other sets of polynomials [5] are seen as special cases of one and the same sequence of set functions, which we have decided to name stochastic sequences of binomial type. Similarly, formerly mysterious computations with Feynman diagrams [1] that went with the integration of products of such polynomials are now seen to reduce to a single identity on the partition lattice.

Our purpose is not only to present a unified theory of known results. It is, rather, to clear the air for the development of further stochastic processes and, we hazard to guess, some rigorous instances of nonlinear quantum field theories. To keep the length of the present paper within bounds, we have left for a later publication the systematic development of chaos theory, the study of new special functions that arise in varying the stochastic measure, as well as the systematization of other notions that are scattered in theories of second quantization, and whose intimate relationship had remained hidden because of the lack of a unifying principle.

We are indebted to D. W. Stroock, who read an earlier draft of the present paper thoroughly and suggested substantial improvements. We also wish to thank P.-A. Meyer, whose recent monograph [25] provided the initial spur to writing this paper.

2. Synopsis. The basic difficulty of stochastic integration is the following. We are given a measure $\phi$ on a set $S$, and we wish to extend such a measure to the product set $S^{n}$. There is a well known and established way of carrying out such an extension, namely, taking the product measure.

While the product measure is adequate in most instances dealing with a scalar valued measure, it turns out to be woefully inadequate when the measure is vector-valued, or, in the case dealt with presently, random-valued. The product measure of a nonatomic scalar measure will vanish on sets supported by lower-dimensional linear subspaces of $S^{n}$. This is not the case, however, for random measures.

The oldest and best known example of this phenomenon is the Gaussian measure. If a measure $\sigma$ is given on the set $S$, then the Gaussian measure $\phi$ is the measure such that, for $\sigma(A)<\infty$, the value $\phi(A)$ is a Gaussian (normal) random variable of mean zero and variance equal to $\sigma(A)$.

The product measure of the Gaussian measure on the set $S^{2}$ does not vanish on the diagonal, that is, on the subset of $S^{2}$ consisting of all points $(s, s)$. In fact, the restriction of such a product measure to the diagonal is none other 
than the measure $\sigma$, as one may verify by an application of the law of large numbers.

The problem therefore arises of modifying the definition of product measure of a random measure in such a way that the resulting measure will vanish on lower-dimensional subsets of $S^{n}$, or diagonal sets, as we call them. This is the problem that is solved by the definition of the stochastic measure. Diagonal sets, that is, subsets of the cube $S^{n}$ consisting of points that have two or more coordinates equal, play a game of musical chairs, which previous workers have managed to avoid by various clever devices. A notable exception is the thesis of Engel [9], whose pioneering work has been the starting point of the present work.

We choose to face directly the combinatorics of the intersection structure of the diagonal sets. This is not hard, once one realizes that the family of diagonal sets is isomorphic to the lattice of partitions of the set $\{1,2, \ldots, n\}$. The sieving out of the overlaps among the various diagonal sets is none other than the Möbius inversion formula on such a lattice of partitions.

After facing up to the need of sieving out overlaps among diagonal sets by the Möbius inversion formula on the lattice of partitions, the definition of a stochastic measure becomes all but trivial, and its properties, classical and new, become a combinatorial game played with partitions.

One may ask for the purpose of defining a stochastic measure, or more precisely, for a natural, or "functorial" definition. The present combinatorial approach leads to the discovery of such a natural definition. In fact, after sieving out all diagonal sets from the set $S^{n}$, all one needs to do is restrict the product measure of $\phi$ to the remaining set, thereby obtaining the stochastic measure. In this way, one sees that the stochastic measure is symmetric under permutations of coordinates, and that furthermore it is supported on the subset of $S^{n}$ consisting of points which are $n$-tuples of distinct coordinates. In other words, the stochastic measure is seen to be the natural measure on $n$-subsets of the set $S$ induced by a given random measure $\phi$.

The bulk of the present work is concerned with the generalization to arbitrary stochastic measures of facts that were previously known in special cases. The celebrated Kailath-Segall [35] formula is given a combinatorial setting that not only substantially generalizes it, but renders it all but obvious.

Perhaps a notable new result in the present work is a formula for the simplified computation of stochastic measures which relies on taking meets of partitions. This universal formula contains as special cases the orthogonality properties of Hermite, Poisson-Charlier and Kravchuk polynomials, as well as all Feynman diagram computations of integrals of products of Hermite polynomials. In fact, it can be viewed as a rigorous version of the method of Feynman diagrams. What is more, the meet formula (Section 5) leads to an extension of the method of Feynman diagrams to the discrete chaos of Wiener and Wintner. We have not developed this line, but we give an example that shows how such an extension might be developed.

Various special polynomial sequences that arose in stochastic integration are here unified under the concept of a stochastic sequence of binomial type. 
Such sequences, giving a very broad generalization of the theory of polynomial sequences of binomial type [32], are ripe objects for further investigation.

A closing word about notation. The symbol $S^{n}$ usually denotes the $n$ dimensional cube of side $S$, that is, the set of all sequences of $n$ entries out of $S$. In other contexts, the symbol $S^{n}$ has been used to denote the set of all functions from the set $[n]=\{1,2, \ldots, n\}$ to the set $S$. In the present work, this ambiguity is avoided. The symbol $S^{b}$ denotes the set of all functions from a subset $b$ of the integers to the set $S$. In particular, the symbol $S^{[n]}$ denotes the set of all functions from the set $[n]$ to the set $S$. One may visualize such functions as sequences, but in the proofs it is preferable to keep in mind that one is dealing with functions.

3. Partitions. We review some basic facts about the lattice of partitions of a finite set $b$, which is denoted by $\Pi(b)[29,30]$. A partition $\sigma$ of $b$ is a family of nonempty disjoint subsets of $b$, called blocks, whose union is the set $b$. The set $\Pi(b)$ is given a partial order by setting $\sigma \leq \pi$ if and only if every block of the partition $\sigma$ is contained in some block of the partition $\pi$. In fact, the partially ordered set $\Pi(b)$ is a lattice, in which the meet of two partitions $\sigma \wedge \pi$ is the partition whose blocks are all the nonempty pairwise intersections of some block of $\pi$ and some block of $\sigma$. Similarly, the join $\sigma \vee \pi$, namely, the smallest partition containing both $\sigma$ and $\pi$ is easily characterized set theoretically.

A segment $[\sigma, \pi]$ of the lattice $\Pi(b)$ is defined as the set of all partitions $\rho$ such that $\sigma \leq \rho \leq \pi$. The zero partition of $\Pi(b)$ (namely, the minimal element of the partially ordered set) is the partition consisting of a separate block for each element; it is denoted by 0 . Similarly, the one partition (namely, the maximum element of the partially ordered set) is the partition consisting of a single block containing all elements; it is denoted by $\hat{1}$.

We will require the Möbius inversion formula on the lattice $\Pi(b)$, and we recall some of the fundamental definitions. The incidence algebra of $\Pi(b)$ is the algebra spanned by all functions $f(\sigma, \pi)$ with values in a field $K$, such that $f(\sigma, \pi)=0$ unless $\sigma \leq \pi$. The sum of any elements of the incidence algebra is formally defined, and the product is defined by convolution, namely,

$$
f * g(\sigma, \pi)=\sum_{\sigma \leq \rho \leq \pi} f(\sigma, \rho) g(\rho, \pi) .
$$

The identity of the incidence algebra is the Kronecker delta: $\delta(\sigma, \pi)=0$ unless $\sigma=\pi$, in which case it equals 1 . The zeta function of the incidence algebra of $\Pi(b)$, in symbols $\zeta(\sigma, \pi)$, is defined to equal 1 if $\sigma \leq \pi$ and 0 otherwise. The Möbius function $\mu(\sigma, \pi)$ is defined to be the inverse of the zeta function in the incidence algebra of $\Pi(b)$, namely, the inverse relative to the convolution product.

If $G$ is a function on the lattice of partitions and

$$
F(\pi)=\sum_{\sigma \geq \pi} G(\sigma)
$$


then the Möbius inversion formula states that

$$
G(\pi)=\sum_{\sigma \geq \pi} \mu(\pi, \sigma) F(\sigma) .
$$

We also have use for partitions $\lambda$ of an integer $n$, which is a distinct concept from partitions of a set, though closely related. We say $\lambda=\left(\lambda_{1}, \ldots, \lambda_{r}\right)$ is a partition of $n$, in symbols $\lambda \vdash n$, if $\lambda_{i} \geq \lambda_{i+1}$ and $\lambda_{1}+\lambda_{2}+\cdots+\lambda_{r}=n$. We also write $\lambda=\left(1^{r_{1}} 2^{r_{2}} \cdots n^{r_{n}}\right)$ to indicate a partition of $n$ in which $r_{i}$ of the $\lambda_{j}$ 's are equal to $i$, for $i=1, \ldots, n$.

The class of a segment $[\sigma, \pi]$, where $\sigma$ and $\pi$ are partitions of a set $b$, is the partition $\left(\lambda_{1}, \ldots, \lambda_{|\pi|}\right)$ of $|\sigma|$ such that $\lambda_{i}$ is the number of blocks of $\sigma$ contained in the $i$ th block of $\pi$. (We may assume the blocks are numbered so that the sequence $\left\{\lambda_{i}\right\}$ is nonincreasing.) Equivalently, it is the partition $\left(1^{r_{1}} 2^{r_{2}} \ldots\right)$ of $|\sigma|$ such that $r_{i}$ blocks of $\pi$ contain exactly $i$ blocks of $\sigma$. We write $r=|\pi|$ and $n=|\sigma|$. Note that

$$
n=\lambda_{1}+\cdots+\lambda_{r}=r_{1}+2 r_{2}+\cdots+n r_{n}
$$

and

$$
r=r_{1}+\cdots+r_{n} .
$$

We sometimes write $\lambda(\sigma, \pi)$ for the class of the segment $[\sigma, \pi]$, and $\lambda(\pi)$ for the class of the segment $[\hat{0}, \pi]$.

It is shown [31] that the Möbius function $\mu(\sigma, \pi)$ depends only on the class of the segment $[\sigma, \pi]$, and that it is given by the following formula:

$$
\mu(\sigma, \pi)=(-1)^{n-r}(2 !)^{r_{3}}(3 !)^{r_{4}} \cdots((n-1) !)^{r_{n}},
$$

where $n=|\sigma|, r=|\pi|$ and $\lambda(\sigma, \pi)=\left(1^{r_{1}} 2^{r_{2}} \cdots n^{r_{n}}\right)$.

We denote by $[n]$ the set $\{1,2, \ldots, n\}$. We write $\mu_{n}$ for $\mu(\hat{0},[n])$. Thus, $\mu_{n}=(-1)^{n-1}(n-1)$ !. The number of partitions of class $\lambda$ of the set $[n]$ is given by

$$
\left[\begin{array}{l}
n \\
\lambda
\end{array}\right]=\frac{n !}{1^{r_{1}} r_{1} ! 2^{r_{2}} r_{2} ! \cdots n^{r_{n}} r_{n} !} .
$$

Let $b_{i}, i=1, \ldots, r$, be the blocks of $\pi$, and let $\lambda_{i}$ be the number of blocks of $\sigma$ contained in the $i$ th block of $\pi$. Then the segment $[\sigma, \pi]$ is isomorphic, as a lattice, to the direct product of the lattices $\left[\hat{0},\left[\lambda_{i}\right]\right], i=1, \ldots, r$. The Möbius function of a direct product lattice is the product of the Möbius function on factors in the product. One easily checks that $(*)$ is consistent with this requirement. (For a treatment of lattices, including a definition of their direct products and Möbius functions, see [30, 31].)

4. Sets and measures. Let $S$ be a set, and $\Sigma$ a $\sigma$-field of subsets of $S$. Elements of $\Sigma$ will be called measurable sets. 
Let $S^{b}$ denote the set of all functions from a finite subset $b$ of positive integers to the set $S$. When $b=[n]$, we sometimes represent such a function from $[n]$ to $S$ as a sequence $\left(s_{1}, \ldots, s_{n}\right)$.

If $b$ is a subset of the positive integers and if $A_{i}, i \in b$, are measurable sets, a rectangle set is the set of functions $f$ from $b$ to $S$ such that $f(i) \in A_{i}$ for all $i \in b$. We indicate this set by the notation $\mathrm{X}_{i \in b} A_{i}$. When $b=[n]$, we also indicate rectangle sets by the notation

$$
A \times B \times \cdots \times C ;
$$

that is, the set of all functions such that $f(1) \in A, f(2) \in B, \ldots, f(n) \in C$.

A subset of $S^{b}$ of the form $A^{b}=A \times \cdots \times A$ ( $|b|$ times), where $A$ is a measurable set in $\Sigma$, is said to be a cubical set. A subset of $S^{b}$ of the form $\mathrm{X}_{i \in b} A_{i}$, where each $A_{i}$ is measurable and where the $A_{i}$ are pairwise disjoint, is said to be a triangular set.

A collection of subsets $\mathscr{E}$ of a set $S$ is a (Bool ean) ring if it contains the empty set and is closed under unions and differences. It is a $\sigma$-ring if it is also closed under countable unions. If a ring ( $\sigma$-ring) is closed under complementation, so that in particular it contains $S$, then it is called a (Boolean) algebra ( $\sigma$ al gebra).

We denote by $\Sigma^{b}$ the set of all rectangle sets in $S^{b}$, by $\Sigma_{\text {fin }}^{\otimes b}$ the Boolean al gebra of subsets of $S^{b}$ generated by all rectangle sets, and by $\Sigma^{\otimes b}$ the Bool ean $\sigma$-al gebra generated by $\Sigma^{b}$. Elements of $\Sigma^{\otimes b}$ will also be called measurable sets.

Every function $f: b \rightarrow S$ has a kernel which is the partition of $b$ whose blocks are defined as follows: $i$ and $j$ of $b$ belong to the same block of the kernel of $f$ whenever $f(i)=f(j)$.

Given a partition $\pi$ of the set $b$ and a set $\mathscr{A}$ in $\Sigma^{\otimes b}$, we denote by $\mathscr{A}_{\pi}$ the set of all functions in $\mathscr{A}$ whose kernel is the partition $\pi$, and we denote by $\mathscr{A}_{(\geq \pi)}$ the set of all functions whose kernel is some partition $\sigma \geq \pi$. A set of the form $\mathscr{A}_{\pi}$ is called a diagonal set. Among all diagonal sets $\mathscr{A}_{\pi}$, the set of the form $\mathscr{A}_{\hat{0}}$ consists of all one-to-one functions, and the set $\mathscr{L}_{\hat{1}}$ consists of all functions which take only one value.

A set of the form $\mathscr{A}_{\hat{1}}$, is called a pure diagonal set. If $\mathscr{A}=A^{b}$, we write $\mathscr{D}^{b}(A)$ in place of $\mathscr{A}_{\hat{1}}$. The set $\left(A^{b}\right)_{\pi}$ is often written $A_{\pi}^{b}$.

The group of permutations of [n] acts on $S^{[n]}$. If $w$ is a permutation of [ $n$ ] and if $\left(s_{1}, \ldots, s_{n}\right)$ is a point of $S^{[n]}$, we set $w\left(s_{1}, \ldots, s_{n}\right)=\left(s_{w(1)}, \ldots, s_{w(n)}\right)$. If $\mathscr{A} \subset S^{[n]}$, we set

$$
w(\mathscr{A})=\left\{w\left(s_{1}, \ldots, s_{n}\right):\left(s_{1}, \ldots, s_{n}\right) \in \mathscr{A}\right\} .
$$

In particular, $w\left(A_{1} \times \cdots \times A_{n}\right)=A_{w(1)} \times \cdots \times A_{w(n)}$.

A subset $\mathscr{A}$ of $\Sigma^{\otimes b}$ is said to be symmetric when $w(\mathscr{A})=\mathscr{A}$ for all permutations $w$ of $b$. The family of all symmetric sets in $\Sigma_{\text {fin }}^{\otimes b}$ is a Bool ean subalgebra of $\Sigma_{\text {fin }}^{\otimes b}$ which will be denoted by $\Sigma_{\text {fin, sym. }}^{\otimes b}$. Similarly, the family of all symmetric sets in $\Sigma^{\otimes b}$ is a Boolean $\sigma$-algebra, which will be denoted by $\Sigma_{\text {sym }}^{\otimes b}$.

Let $\phi$ be a set function on some ring of sets $\mathscr{R}$, taking values in a Banach space $X$. We say that $\phi$ is finitely additive if $\phi(\varnothing)=0$ and if, whenever 
$A=\bigcup_{i=1}^{m} A_{i}$, where the $A_{i}$ are disjoint sets in $\mathscr{R}$,

$$
\phi(A)=\sum_{i=1}^{m} \phi\left(A_{i}\right)
$$

We say that $\phi$ is (norm) countably additive if $\phi(\varnothing)=0$ and if, whenever $A=\cup_{i=1}^{\infty} A_{i}$, where the $A_{i}$ are disjoint elements of $\mathscr{R}$,

$$
\lim _{m \rightarrow \infty} \phi\left(\bigcup_{i=1}^{m} A_{i}\right)=\phi(A)
$$

where the limit is taken in $X$.

A vector-valued measure on $\mathscr{R}$ is a norm countably additive set function on $\mathscr{R}$ taking values in a Banach space $X$. Finitely additive vector-valued measures are defined analogously.

Let $(\Omega, \mathscr{P}, P)$ be a probability space, and let $\mathscr{M}$ be the space of real-valued measurable functions on $(\Omega, \mathscr{P}, P)$, that is, real random variables. If $f$ and $g$ are in $\mathscr{M}$, we define their product $f g$ by

$$
(f g)(\omega)=f(\omega) g(\omega), \quad \omega \in \Omega .
$$

The expectation of a random variable $f$ is denoted $\langle f\rangle$.

A random measure is a vector-valued measure taking values in a Banach space $X$ of random variables; for example, $X=L^{2}(\Omega, \mathscr{P}, P)$. The space $X$, as a subspace of $\mathscr{M}$, inherits its multiplicative structure. Note, however, that $f g$ need not belong to $X$, and a fortiori, multiplication need not be continuous. Thus, $X$ is not a Banach algebra, but rather a Banach space equipped with a not-everywhere-defined multiplicative structure.

If $\phi$ is a random-valued measure, we write $\langle\phi\rangle$ for the real measure given by $\langle\phi\rangle(A)=\langle\phi(A)\rangle$.

We next consider products of measures. A set function $\nu\left(A_{1}, A_{2}, \ldots, A_{n}\right)$, where the $n$ variables $A_{1}, A_{2}, \ldots, A_{n}$ range over $\Sigma$, is called a multimeasure if it is a measure in each variable when each of the other variables is held fixed. Given an $X$-valued multimeasure $\nu$ on $\Sigma^{[n]}$, let $\mathscr{R}_{\nu}$ be the ring generated by the rectangles

$$
\left\{\mathscr{A} \in \Sigma^{[n]}: \nu\left(A_{1}, \ldots, A_{n}\right) \in X\right\} .
$$

Then there exists a unique finitely additive measure $m_{n}$ on $\mathscr{R}_{\nu}$ such that

$$
m_{n}\left(A_{1} \times \cdots \times A_{n}\right)=\nu\left(A_{1}, \ldots, A_{n}\right) .
$$

The measure $m_{n}$ will be said to be associated to the multimeasure $\nu$.

The ordinary notion of product of two measures $\eta$ and $\psi$ can be defined by means of a multimeasure. Let $b=\left\{i_{1}, \ldots, i_{k}\right\}$ be a subset of $[n]$, with $i_{1}<$ $\cdots<i_{k}$ and let $b^{c}=\left\{j_{1}, \ldots, j_{n-k}\right\}$ with $j_{1}<\cdots<j_{n-k}$ be the complement of $b$. A multimeasure $\nu$ is defined on rectangular sets by setting

$$
\nu\left(A_{1}, \ldots, A_{n}\right)=\eta\left(A_{i_{1}} \times \cdots \times A_{i_{k}}\right) \psi\left(A_{j_{1}} \times \cdots \times A_{j_{n-k}}\right) .
$$


The finitely additive measure on $\mathscr{R}_{\nu}$ associated with $\nu$ will be denoted $\eta^{b} \otimes \psi^{b^{c}}$. A similar construction may and will be used to define the product of more than two measures.

We will assume from now on that all diagonal sets belong to $\Sigma^{\otimes[n]}$. We will also assume that $\phi$ is a random measure, taking values in a Banach space of random variables $X$. Finally, we assume that all finitely additive product measures, defined in terms of an associated multimeasure as above, have unique countably additive extensions to some ring containing $\mathscr{R}_{\nu}$, where $\mathscr{R}_{\nu}$ is defined above. We will say that the random measure $\phi$ is good (for lack of a better term) when the space $(S, \Sigma)$ and the process $\phi$ satisfy these assumptions.

The assumption that the diagonals are contained in the product $\sigma$-algebra is satisfied when $S$ is a complete metrizable separable space, that is, a Polish space, with $\Sigma$ the corresponding Borel algebra.

The proof that certain finitely additive product measures have unique countably additive extensions is given by Engel [9]. Engel's results can be used to establish the existence of a product measure for measures defined from multimeasures as above. Our examples satisfy Engel's assumptions, and thus deal with countably additive measures. In particular, we have benefited from Engel's ideas in the proof of Proposition 12.

5. Stochastic measures. We repeat the definitons of $\mathscr{A}_{(\geq \pi)}$ and $\mathscr{A}_{\pi}$ in slightly different language, when $b=[n]$. Write $i \sim_{\pi} j$ when $i$ and $j$ are in the same block of $\pi$. Then

$$
\mathscr{A}_{(\geq \pi)}=\left\{\left(s_{1}, s_{2}, \ldots, s_{n}\right) \in \mathscr{A}: s_{i}=s_{j} \text { if } i \sim_{\pi} j\right\}
$$

and

$$
\mathscr{A}_{\pi}=\left\{\left(s_{1}, s_{2}, \ldots, s_{n}\right) \in \mathscr{A}: s_{i}=s_{j} \text { if and only if } i \sim_{\pi} j\right\} .
$$

We stress the facts that, in both cases, the points $s_{i}$ and $s_{j}$ coincide whenever $i$ and $j$ are in the same block $b$ of $\pi$, but in the definition of $\mathscr{A}_{\pi}$ we require in addition that $s_{i}$ and $s_{j}$ shall not coincide if $i$ and $j$ are in different blocks of $\pi$.

The following trivial partition of $\mathscr{A}_{(\geq \pi)}$ is fundamental:

$$
\mathscr{A}_{(\geq \pi)}=\bigcup_{\sigma \geq \pi} \mathscr{A}_{\sigma} \quad \text { (disjoint union). }
$$

Definition 1. Let $\phi$ be a good random measure on $(S, \Sigma)$. Define, for $\mathscr{A} \in$ $\Sigma^{\otimes b}$, and for $\pi$ a partition of the set $b$,

$$
\phi_{\pi}^{b}(\mathscr{A})=\left(\bigotimes_{i \in b} \phi\right)\left(\mathscr{A}_{(\geq \pi)}\right)
$$

and

$$
\mathrm{St}_{\pi}^{b}(\mathscr{A})=\left(\bigotimes_{i \in b} \phi\right)\left(\mathscr{A}_{\pi}\right)
$$


Neither $\phi_{\pi}^{b}$ nor $\mathrm{St}_{\pi}^{b}$ is defined unless $\pi$ is a partition of the set $b$. Since $\mathscr{A}_{(\geq 0)}=\mathscr{A}$, we have

$$
\phi_{0}^{b}=\bigotimes_{i \in b} \phi .
$$

The product measure is $\phi_{\hat{0}}^{b}$. We will consistently use this notation for product measures.

The following proposition is fundamental.

Proposition 1. The measures $\phi_{\pi}^{b}$ and $\mathrm{St}_{\pi}^{b}$ are related as follows:

$$
\begin{aligned}
\phi_{\pi}^{b} & =\sum_{\sigma \geq \pi} \mathrm{St}_{\sigma}^{b}, \\
\mathrm{St}_{\pi}^{b} & =\sum_{\sigma \geq \pi} \mu(\pi, \sigma) \phi_{\sigma}^{b} .
\end{aligned}
$$

Proof. The first equation follows directly from $(*)$. The second follows from Möbius inversion.

The following special case of the preceding proposition is one of our main results.

THEOREM 1. For $\pi=\hat{0}$ and $b=[n]$, we have the identity

$$
\mathrm{St}_{\hat{0}}^{[n]}=\sum_{\sigma \geq \hat{0}} \mu(\hat{0}, \sigma) \phi_{\sigma}^{[n]} \text {. }
$$

The measure $\mathrm{St}_{\hat{0}}^{[n]}$ will be called the stochastic measure of degree $n$, or, when no confusion is possible, the stochastic measure associated to the measure $\phi$.

An integral

$$
\int f\left(s_{1}, \ldots, s_{n}\right) \operatorname{St}_{\hat{0}}^{[n]}\left(d s_{1} \cdots d s_{n}\right)
$$

is said to be an $n$th multiple stochastic integral or simply, when no confusion may arise, the stochastic integral of the function $f$. It is evident from its definition that $\mathrm{St}_{\hat{0}}^{[n]}$ is symmetric. That is,

$$
\mathrm{St}_{\hat{0}}^{[n]}(w(\mathscr{A}))=\mathrm{St}_{\hat{0}}^{[n]}(\mathscr{A}),
$$

where $w$ is a permutation of the set $[n]$.

As already remarked, the measure $\phi_{\hat{0}}^{[n]}$ is a product measure. We will now see that, more generally, the measure $\phi_{\pi}^{[n]}$ is expressible as the product over the blocks $b$ of $\pi$ of the measures $\phi_{\hat{1}}^{b}$.

Proposition 2. The measure $\phi_{\pi}^{[n]}$ is a product measure:

$$
\phi_{\pi}^{[n]}=\bigotimes_{b \in \pi} \phi_{\hat{1}}^{b} .
$$


PRoOF. Both measures are the restriction of the product measure $\phi_{\hat{0}}^{[n]}$ to the set of elements of $S^{[n]}$ whose kernel $\sigma$ is greater than or equal to $\pi$.

The measures $\phi_{\hat{1}}^{b}$ are concentrated on the pure diagonal sets $\mathscr{A}_{\hat{1}}$ :

$$
\phi_{\hat{1}}^{b}(\mathscr{A})=\phi_{\hat{0}}^{b}\left(\mathscr{A}_{\hat{1}}\right) \text {. }
$$

If the set $b$ has a single el ement, then $\phi_{\hat{1}}^{b}=\phi$. Another immediate consequence of the definitions is the identity $\mathrm{St}_{\hat{1}}^{b}=\phi_{\hat{1}}^{b}$.

We have shown that $\mathrm{St}_{\pi}^{[n]}$ could be expressed as the Möbius sum of measures $\phi_{\sigma}^{[n]}$. We now show that $\mathrm{St}_{\pi}^{[n]}$ can also be computed by a simple recursion over products of measures $\phi_{\hat{1}}^{b}$ and lower order stochastic measures. The next theorem is a generalization of a formula first given by Kailath and Segall [35]. We shall see that it can be applied to derive recursion relations for orthogonal polynomials.

THEOREM 2. The following identity holds on $\Sigma^{\otimes[n]}$ :

$$
\mathrm{St}_{\hat{0}}^{[n]}=\sum \mu_{|b|} \phi_{\hat{1}}^{b} \otimes \mathrm{St}_{\hat{0}}^{b^{c}},
$$

where the sum ranges over all subsets $b$ of $[n]$ containing the element 1 .

Proof. By $(* *)$,

$$
\mathrm{St}_{\hat{0}}^{[n]}=\sum_{\pi} \mu(\hat{0}, \pi) \phi_{\pi}^{[n]}
$$

Given a partition $\pi$ of $[n]$, let $b$ be the block of $\pi$ containing the element 1 , and let $\pi^{\prime}$ be the partition of $b^{c}$ consisting of the remaining blocks of $\pi$. Now $\phi_{\pi}^{[n]}=\phi_{\hat{1}}^{b} \otimes \phi_{\pi^{\prime}}^{b^{c}}$, and $\mu(\hat{0}, \pi)=\mu_{|b|} \mu\left(\hat{0}, \pi^{\prime}\right)$. Therefore,

$$
\begin{aligned}
\sum_{\pi} \mu(\hat{0}, \pi) \phi_{\pi}^{[n]} & =\sum_{b} \sum_{\pi^{\prime} \in \Pi\left(b^{c}\right)} \mu_{|b|} \mu\left(\hat{\mathrm{O}}, \pi^{\prime}\right) \phi_{\hat{1}}^{b} \otimes \phi_{\pi^{\prime}}^{b^{c}} \\
& =\sum_{b} \mu_{|b|} \phi_{\hat{1}}^{b} \otimes \mathrm{St}_{\hat{0}}^{b^{c}} .
\end{aligned}
$$

Proposition 1 represents $S t_{\hat{o}}^{b}$ as a linear combination of the measures $\phi_{\pi}^{b}$, where the sum extends over all partitions $\pi$. We next show that the product of stochastic measures can also be expressed as a linear combination of the measures $\phi_{\pi}^{b}$, where the sum extends over all partitions in an appropriate segment.

Theorem 3. Let $\tau$ be a partition of $[n]$. Then

$$
\bigotimes_{b \in \tau} \mathrm{St}_{\hat{0}}^{b}=\sum_{\hat{0} \leq \pi \leq \tau} \mu(\hat{0}, \pi) \phi_{\pi}^{[n]} .
$$


PRoOF. If $b$ is a block of $\tau$, and $\tau^{\prime}$ is the partition on $b^{c}$ consisting of remaining blocks of $\tau$, then

$$
\begin{aligned}
\sum_{\hat{0} \leq \pi \leq \tau} \mu(\hat{0}, \pi) \phi_{\pi}^{b} & =\left(\sum_{\rho \in \Pi(b)} \mu(\hat{0}, \rho) \phi_{\rho}^{b}\right) \otimes\left(\sum_{\hat{0} \leq \pi^{\prime} \leq \tau^{\prime}} \mu\left(\hat{0}, \pi^{\prime}\right) \phi_{\pi^{\prime}}^{b^{c}}\right) \\
& =\mathrm{St}_{\hat{0}}^{b} \otimes\left(\sum_{\hat{0} \leq \pi^{\prime} \leq \tau^{\prime}} \mu\left(\hat{0}, \pi^{\prime}\right) \phi_{\pi^{\prime}}^{b^{c}}\right) .
\end{aligned}
$$

The conclusion follows by induction.

The product of stochastic measures may also be expressed as a sum over measures $\mathrm{St}_{\sigma}^{[n]}$. The following result is fundamental in the combinatorial interpretation of orthogonal polynomials.

TheOREM 4. Let $\pi$ be a partition of $[n]$. Then

$$
\bigotimes_{b \in \pi} \mathrm{St}_{\hat{0}}^{b}=\sum_{\{\sigma: \sigma \wedge \pi=\hat{0}\}} \mathrm{St}_{\sigma}^{[n]} .
$$

Proof. By Theorem 3,

$$
\bigotimes_{b \in \pi} \mathrm{St}_{\hat{0}}^{b}=\sum_{\hat{0} \leq \sigma \leq \pi} \mu(\hat{0}, \sigma) \phi_{\sigma}^{[n]} .
$$

But

$$
\phi_{\sigma}^{[n]}=\sum_{\tau \geq \sigma} \mathrm{St}_{\sigma}^{[n]} .
$$

Therefore,

$$
\begin{aligned}
\bigotimes_{b \in \pi} \mathrm{St}_{\hat{0}}^{b} & =\sum_{\hat{0} \leq \sigma \leq \pi} \mu(\hat{0}, \sigma) \sum_{\tau \geq \sigma} \mathrm{St}_{\tau}^{[n]} \\
& =\sum_{\sigma \geq \hat{0}} \mu(\hat{0}, \sigma) \zeta(\sigma, \pi) \sum_{\tau \geq \hat{0}} \zeta(\sigma, \tau) \mathrm{St}_{\tau}^{[n]} \\
& =\sum_{\tau \geq \hat{0}} \mathrm{St}_{\tau}^{[n]} \sum_{\sigma \geq \hat{0}} \mu(\hat{0}, \sigma) \zeta(\sigma, \pi \wedge \tau) \\
& =\sum_{\tau \geq \hat{0}} \mathrm{St}_{\tau}^{[n]} \sum_{\hat{0} \leq \rho \leq(\pi \wedge \tau)} \mu(\hat{0}, \rho) \\
& =\sum_{\{\tau: \pi \wedge \tau=\hat{0}\}} \mathrm{St}_{\tau}^{[n]} .
\end{aligned}
$$

The final equality follows from the fact that the sum of the Möbius function over a segment $[\sigma, \pi]$ is zero unless $\sigma=\pi$, in which case it equals one. 
We shall now state a property which characterizes a stochastic measure associated with a given measure $\phi$. We begin with a preliminary definition.

Recall that a measurable set $A_{1} \times \cdots \times A_{n} \in \Sigma^{[n]}$ is triangular when the measurable sets $A_{1}, \ldots, A_{n}$ in $\Sigma$ are pairwise disjoint. If $\mathscr{A}=A_{1} \times \cdots \times A_{n}$ is a triangular set, then as $w$ ranges over all permutations of $[n]$, the family of $n$ ! sets $A_{w(1)} \times \cdots \times A_{w(n)}$ consists of pairwise disjoint sets. Hence, the set $\bigcup_{w} w(\mathscr{A})$ belongs to $\Sigma_{\text {fin }}^{\otimes[n]}$. We denote this set by perm $\left(A_{1} \times \cdots \times A_{n}\right)$. Note that $\operatorname{perm}\left(A_{1} \times \cdots \times A_{n}\right)$ is not defined unless the set $A_{1} \times \cdots \times A_{n}$ is triangular.

The family of all sets of the form perm $\left(A_{1} \times \cdots \times A_{n}\right)$ is closed under unions and intersections (but not under complements). In other words, it is a distributive lattice of sets.

Proposition 3. The $\sigma$-field generated by sets of the form perm $\left(A_{1} \times \cdots \times\right.$ $A_{n}$ ), where $A_{1} \times \cdots \times A_{n}$ is triangular coincides with the $\sigma$-field of all symmetric sets contained in $S_{\hat{0}}^{[n]}$.

The proof is omitted.

We are now ready to give an intrinsic characterization of the stochastic measure $\mathrm{St}_{\hat{0}}^{[n]}$ associated to a random measure $\phi$.

THEOREM 5. For a given countably additive random-valued good measure $\phi$ on $(S, \Sigma)$, there exists one and only one measure $\mathrm{St}_{0}^{[n]}$ on symmetric sets contained in $S_{\hat{0}}^{[n]}$, such that

$$
\mathrm{St}_{\hat{0}}^{[n]}\left(\operatorname{perm}\left(A_{1} \times \cdots \times A_{n}\right)\right)=n ! \phi\left(A_{1}\right) \cdots \phi\left(A_{n}\right) .
$$

PRoOF. In view of the preceding proposition, such a measure is unique if it exists. I ts existence is guaranteed by the Möbius inversion formula

$$
\mathrm{St}_{\hat{0}}^{[n]}=\sum_{\pi} \mu(\hat{0}, \pi) \phi_{\pi}^{[n]} .
$$

Indeed, since $A_{1} \times \cdots \times A_{n}$ is triangular, $\left(A_{1} \times \cdots \times A_{n}\right)_{\pi}=\varnothing$ unless $\pi=\hat{0}$. Thus $\phi_{\pi}^{[n]}\left(A_{1} \times \cdots \times A_{n}\right)=0$ unless $\pi=\hat{0}$. When $\pi=\hat{0}$,

$$
\phi_{\hat{0}}^{[n]}\left(\operatorname{perm}\left(A_{1}, \ldots, A_{n}\right)\right)=n ! \phi\left(A_{1}\right) \times \cdots \times \phi\left(A_{n}\right) .
$$

6. Stochastic sequences of binomial type. A sequence of measures $\psi_{n}$ on $(S, \Sigma)$ may be defined by setting $\psi_{n}(A)$ to equal the stochastic measure $\operatorname{St}_{\hat{0}}^{[n]}$ of cubical set $A^{[n]}$. The stochastic measure of any set can then be approximated by sums of products of the $\psi_{n}$. The $\psi_{n}(A)$, furthermore, are of binomial type (v. below, Theorem 6), and special cases of these sequences are sequences of orthogonal polynomials (Section 7).

DeFinition 2. Given a good random measure $\phi$ on $(S, \Sigma)$ we define the stochastic sequence of binomial type $\psi_{n}(A), n=1,2, \ldots$, to be the sequence of 
random-valued set functions

$$
\psi_{n}(A)=\mathrm{St}_{\hat{0}}^{[n]}\left(A^{[n]}\right)
$$

for all $A \in \Sigma$ of finite measure. form

Proposition 4. If $\mathscr{A} \in \Sigma_{\text {fin }}^{\otimes[n]}$, then $\operatorname{St}_{\hat{0}}^{[n]}(\mathscr{A})$ is the sum of products of the

$$
\psi_{i_{1}}\left(A_{1}\right) \cdots \psi_{i_{r}}\left(A_{r}\right)
$$

where $i_{1}+\cdots+i_{r}=n$, and the sets $A_{1}, \ldots, A_{r}$ are disjoint.

Proof. Any set $\mathscr{A} \in \Sigma_{\text {fin }}^{\otimes[n]}$ is the finite union of disjoint rectangles. It can easily be shown that any rectangle can be expressed as the disjoint union of rectangles whose sides are pairwise equal or disjoint. Let $\mathscr{A}=A_{1} \times \cdots \times A_{n}$ be such a rectangle, considered as a function from $[n]$ to $\Sigma$, and let $\tau$ be the kernel of $\mathscr{A}$. That is, if $b$ is a block of $\tau$, then $A_{i}=A_{j}$ for any two elements $i$ and $j$ of $b$; we denote this common value by the symbol $A_{b}$. Conversely, if $i$ and $j$ are not in the same block of $\tau$, then $A_{i}$ and $A_{j}$ are disjoint. If $\sigma>\tau$, then $\mathscr{A}_{\sigma}=\varnothing$, and $\phi_{\sigma}^{[n]}(\mathscr{A})=\phi_{0}^{[n]}\left(\mathscr{A}_{(\geq \sigma)}\right)=0$. Thus, by Theorem 3,

$$
\begin{aligned}
\mathrm{St}_{\hat{0}}^{[n]}(\mathscr{A}) & =\sum_{\pi \geq \hat{0}} \mu(\hat{0}, \pi) \phi_{\hat{0}}^{[n]}(\mathscr{A}) \\
& =\sum_{\hat{0} \leq \pi \leq \tau} \mu(\hat{0}, \pi) \phi_{\hat{0}}^{[n]}(\mathscr{A}) \\
& =\bigotimes_{b \in \tau} \mathrm{St}_{\hat{0}}^{b}(\mathscr{A}) \\
& =\prod_{b \in \tau} \psi_{|b|}\left(A_{b}\right) .
\end{aligned}
$$

The following theorem is fundamental and justifies our terminology.

THEOREM 6. If $\psi_{n}(A)$ is the stochastic sequence of binomial type associated with a measure $\phi$ on a good measure space $(S, \Sigma, \sigma)$, and if $A$ and $B$ are disjoint sets in $\Sigma$ of finite measure, we have

$$
\psi_{n}(A \cup B)=\sum_{k=0}^{n}\left(\begin{array}{l}
n \\
k
\end{array}\right) \psi_{k}(A) \psi_{n-k}(B) .
$$

Proof.

$$
\mathrm{St}_{\hat{0}}^{[n]}\left((A \cup B)^{[n]}\right)=\sum_{b \subset[n]} \mathrm{St}_{\hat{0}}^{[n]}\left(A^{b} \times B^{b^{c}}\right) .
$$

A typical summand on the right-hand side is

$$
\mathrm{St}_{\hat{0}}^{[n]}\left(A^{b} \times B^{b^{c}}\right)=\sum_{\pi \geq \hat{0}} \mu(\hat{0}, \pi) \phi_{\pi}^{[n]}\left(A^{b} \times B^{b^{c}}\right) .
$$


As in the previous proof, $\phi_{\pi}^{[n]}\left(A^{b} \times B^{b^{c}}\right)=0$ if $\pi>\tau$, where $\tau$ is the partition $\left\{b, b^{c}\right\}$. The Möbius sum thus terminates at $\pi=\tau$, and by Theorem 3,

$$
\mathrm{St}_{\hat{0}}^{[n]}\left(A^{b} \times B^{b^{c}}\right)=\mathrm{St}_{\hat{0}}^{b}\left(A^{b}\right) \mathrm{St}_{\hat{0}}^{b^{c}}\left(B^{b^{c}}\right) .
$$

But if $|b|=k$,

$$
\mathrm{St}_{\hat{0}}^{b}\left(A^{b}\right)=\mathrm{St}_{\hat{0}}^{[k]}\left(A^{[k]}\right),
$$

and similarly for $\mathrm{St}_{\hat{0}}^{b^{c}}$. There are $\left(\begin{array}{l}n \\ k\end{array}\right)$ partitions of $[n]$ into blocks $\left\{b, b^{c}\right\}$ with $|b|=k$.

In a similar way, one may prove a multinomial expansion.

Proposition 5. If $A=\bigcup_{i=1}^{r} A_{i}$ and the $A_{i}$ are pairwise disjoint, then

$$
\psi_{n}(A)=\sum_{i_{1}+\cdots+i_{r}=n}\left(\begin{array}{c}
n \\
i_{1} \cdots i_{r}
\end{array}\right) \psi_{i_{1}}\left(A_{1}\right) \cdots \psi_{i_{r}}\left(A_{r}\right) .
$$

Useful as the measures $\psi_{n}$ are, we require some further measures, namely the diagonal measures $\Delta_{n}$. These are defined in terms of the measures $\phi_{\hat{1}}^{[n]}$.

Definition 3. For every positive integer $n$, the $n$th diagonal measure $\Delta_{n}$ of $\phi$ is the measure on $(S, \Sigma)$ given by

$$
\Delta_{n}(A)=\phi_{\hat{1}}^{[n]}\left(A^{[n]}\right)=\mathrm{St}_{\hat{1}}^{[n]}\left(A^{[n]}\right)
$$

We omit the verification that $\Delta_{n}$ is a measure.

Theorem 2 implies the following recursion relation for the measures $\psi_{n}$.

THEOREM 7. If $\psi_{n}(A)$ is the stochastic sequence of binomial type associated with the random measure $\phi$, then

$$
\psi_{n}(A)=\sum_{k=1}^{n}(-1)^{k-1}(n-1)_{k-1} \Delta_{k}(A) \psi_{n-k}(A) .
$$

Theorem 1 implies the following expression for $\psi_{n}$ in terms of the diagonal measures:

THEOREM 8.

$$
\psi_{n}(A)=\sum_{\lambda \vdash n}\left(\begin{array}{c}
n \\
r_{1} \cdots r_{n}
\end{array}\right) \frac{(-1)^{r_{1}+\cdots+r_{n}-n}}{\prod_{i=1}^{n} i^{r_{i}}} \Delta_{1}^{r_{1}}(A) \cdots \Delta_{n}^{r_{n}}(A),
$$

where $\lambda=\left(1^{r_{1}} 2^{r_{2}} \cdots n^{r_{n}}\right)$.

The proof is omitted.

Given any partition $\lambda \vdash n$, one can define, in analogy to $\psi_{n}$, the measure

$$
\Psi_{\lambda}^{n}(A)=\mathrm{St}_{\pi}^{[n]}\left(A^{[n]}\right),
$$


where $\pi$ is any partition of class $\lambda$. The measure $\Psi_{\lambda}^{n}$ is well defined, by virtue of the symmetry of the argument $A^{[n]}$. The measures $\Psi_{\lambda}^{n}$ are of independent interest, and hint at a rich theory which extends the theory of orthogonal polynomials.

7. Examples. In the following examples, all random measures are assumed to be good.

EXAMPLE D (Discrete). Let $S$ be a set, and let $\Sigma$ be the $\sigma$-algebra generated by all singletons $\{s\}$. Suppose that the measure $\phi$ is so defined that $\phi(s)$ equals either 0 or 1 for every element $s \in S$, and suppose that $\phi(S)<\infty$. Then for every measurable function $f\left(s_{1}, \ldots, s_{n}\right)$ we have

$$
\int f\left(s_{1}, \ldots, s_{n}\right) \operatorname{St}_{\hat{0}}^{[n]}\left(d s_{1}, \ldots, d s_{n}\right)=\sum f\left(s_{1}, \ldots, s_{n}\right),
$$

where the sum ranges over all sequences $\left(s_{1}, \ldots, s_{n}\right)$ of elements of $S$ all of whose entries are distinct. The sequence of binomial type associated with the discrete measure is

$$
\psi_{n}(A)=(|A|)_{n},
$$

where $(x)_{n}=x(x-1) \cdots(x-n+1)$ is the falling factorial.

The diagonal measures are given by

$$
\Delta_{n}(A)=|A|, \quad n \geq 1 .
$$

EXAMPLE SP (Symmetric polynomials) [8]. Let $\Sigma$ be the Bool ean algebra of all subsets of a set $S$. Let $Z[S]$ be the polynomial ring with integer coefficients in the set of variables $S$. Define a measure $\phi$ as follows: for every finite subset $A$ of $S$,

$$
\phi(A)=\sum_{a \in A} a .
$$

Set $p_{n}(A)=\sum_{a \in A} a^{n}$, and for every partition $\pi$ of $[n]$, set $p_{\pi}(A)=\prod_{b \in \pi} p_{|b|}$. The polynomials $p_{n}(A)$ are known as the power sum symmetric polynomials.

One verifies that $\phi_{\pi}^{[n]}\left(A^{n}\right)=p_{\pi}(A)$, and $\psi_{n}(A)=n ! e_{n}(A)$, where $e_{n}$ is the $n$th elementary symmetric polynomial:

$$
e_{n}(A)=\sum_{i_{1}<i_{2}<\cdots<i_{n}} a_{i_{1}} a_{i_{2}} \cdots a_{i_{n}},
$$

where $\left(a_{1}, a_{2}, \ldots\right)$ is some linear ordering of the elements of $A$.

The symmetric polynomials $\mathrm{St}_{\pi}^{[n]}\left(A^{n}\right)$ are known as the monomial symmetric polynomials, and are denoted by $m_{\pi}(A)=m_{\pi}\left(a_{1}, a_{2}, \ldots\right)$.

The diagonal measures are given by

$$
\Delta_{n}(A)=\sum_{a \in A} a^{n}
$$


EXAMPLE G (Gaussian measure). Let $\sigma$ be a nonatomic real measure on $(S, \Sigma)$, and let $\phi$ be an $L^{2}$ random-valued measure on $(S, \Sigma)$ such that for any set $A \in \Sigma$ with $\sigma(A)$ finite, $\phi(A)$ is a Gaussian random variable with mean zero and variance $\sigma(A)$. Furthermore, assume that if $\left\{A_{i}\right\}$ is a set of pairwise disjoint elements of $\Sigma$ with $\sigma(A)$ finite, then the random variables $\left\{\phi\left(A_{i}\right)\right\}$ are independent.

The stochastic sequence of binomial type associated with the Gaussian measure is the sequence

$$
\psi_{n}(A)=H_{n}^{(v)}(\phi(A))
$$

where $v=\sigma(A)$. The polynomials $H_{n}^{(v)}(x)$ are the Hermite polynomials of variance $v$ in the variable $x$. We take the preceding equation as the definition of the Hermite polynomials; all properties of the Hermite polynomials are consequences of this definition. For example, we have the identity ([15], [32], page 722)

$$
H_{n}^{(v+w)}(x+y)=\sum_{k \geq 0}\left(\begin{array}{l}
n \\
k
\end{array}\right) H_{k}^{(v)}(x) H_{k}^{(w)}(y) .
$$

We next compute the diagonal measures for the Gaussian process, using Proposition 12 of Section 9.

Proposition 6. The diagonal measures of the Gaussian measure are given by

$$
\begin{aligned}
& \Delta_{2}(A)=\sigma(A), \\
& \Delta_{n}(A)=0, \quad n>2 .
\end{aligned}
$$

Proof. For every positive integer $N$, let $\left\{A_{N i}, i=1, \ldots, N\right\}$ be a collection of disjoint sets such that $\cup_{i=1}^{N} A_{N i}=A$ and $\sigma\left(A_{N i}\right)=\sigma(A) / N$. Proposition 12 gives conditions under which

$$
\Delta_{n}(A)=\lim _{N \rightarrow \infty} \sum_{i=1}^{N} \phi\left(A_{N i}\right)^{n}
$$

in $L^{2}$. However,

$$
\sum_{i=1}^{N}\left[\phi\left(A_{N i}\right)\right]^{n}=\frac{1}{N^{n / 2-1}}\left(\frac{1}{N} \sum_{i=1}^{N}\left(\sqrt{N} \phi\left(A_{N i}\right)\right)^{n}\right) .
$$

The Gaussian random variables $\sqrt{N} \phi\left(A_{N i}\right)$ are independent and each one has variance $\sigma(A)$. The term in parentheses is finite in $L^{2}$ for any $n>1$. For $n>2$, therefore, the right-hand side converges to zero as $m \rightarrow \infty$. When $n=2$, it converges to $\sigma(A)$ by the law of large numbers. The hypotheses of Proposition 12 are easily verified. 
Theorem 7 now gives the classical recursion formula for the Hermite polynomials:

$$
H_{n}^{(v)}(x)=x H_{n-1}^{(v)}(x)-(n-1) v H_{n-2}^{(v)}(x) .
$$

Theorem 8 implies the following known expression for $H_{n}^{(v)}(x)$ [19]:

$$
H_{n}^{(v)}(x)=\sum_{j=0}^{\lfloor n / 2\rfloor} x^{n-2 j}(-v)^{j} c(n, j),
$$

where

$$
c(n, j)=\frac{n !}{(n-2 j) !(2 j) ! !},
$$

and $\lfloor n / 2\rfloor$ is the largest integer less than $n / 2$.

EXAMPLE P (Poisson). Let $\sigma$ be a nonatomic measure on $(S, \Sigma)$. Let $\phi$ be the random process such that, for every measurable set $A$, the random variable $\phi(A)$ has a Poisson distribution with parameter $\sigma(A)$, and such that if $\left\{A_{i}\right\}$ is a set of pairwise disjoint sets, then $\left\{\phi\left(A_{i}\right)\right\}$ is a set of independent random variables.

Proposition 7. The diagonal measures of the Poisson process are

$$
\Delta_{n}(A)=\phi(A), \quad n=1,2, \ldots
$$

Proof. Let $\left\{A_{N i}\right\}$ be as above, with $\sigma$ now the (nonatomic) measure defining the Poisson process. We can thus use Proposition 12 to calculate $\Delta_{n}(A)$ as $\sum_{i=1}^{N} \phi\left(A_{N i}\right)^{n}$. But

$$
\begin{aligned}
\left\|\sum_{i=1}^{N} \phi\left(A_{N i}\right)^{n}-\phi(A)\right\|^{2}= & \sum_{i=1}^{N}\left\|\phi\left(A_{N i}\right)^{n}-\phi\left(A_{N i}\right)\right\|^{2} \\
& +\sum_{i \neq j}\left\langle\left(\phi\left(A_{N i}\right)^{n}-\phi\left(A_{N i}\right)\right)\left(\phi\left(A_{N j}\right)^{n}-\phi\left(A_{N j}\right)\right)\right\rangle
\end{aligned}
$$

To estimate these terms, recall that for the Poisson process,

$$
P(\{\phi(A)=j\})=\frac{\sigma(A)^{j} e^{-\sigma(A)}}{j !} .
$$

Thus,

$$
\begin{aligned}
\left\|\phi\left(A_{N i}\right)^{n}-\phi\left(A_{N i}\right)\right\|^{2} & =\sum_{j=0}^{\infty} \frac{\left(j^{n}-j\right)^{2}}{j !}\left(\frac{\sigma(A)}{N}\right)^{j} e^{-\sigma(A) / N} \\
& =\frac{1}{N^{2}} \sum_{j=2}^{\infty} \frac{\left(j^{n}-j\right)^{2}}{j !} \frac{\sigma(A)^{j}}{N^{j-2}} e^{-\sigma(A) / N} \\
& \leq \frac{C_{1}}{N^{2}}
\end{aligned}
$$


Similarly,

$$
\begin{aligned}
& \left\langle\left(\phi\left(A_{N i_{1}}\right)^{n}-\phi\left(A_{N i_{1}}\right)\right)\left(\phi\left(A_{N i_{2}}\right)^{n}-\phi\left(A_{N i_{2}}\right)\right)\right\rangle \\
& \quad=\sum_{j, k=0}^{\infty} \frac{\left(j^{n}-j\right)\left(k^{n}-k\right)}{j ! k !}\left(\frac{\sigma(A)}{N}\right)^{j+k} e^{-2 \sigma(A) / N}=\frac{C_{2}}{N^{4}} .
\end{aligned}
$$

Thus,

$$
\left\|\sum_{i=1}^{N} \phi\left(A_{N i}\right)^{n}-\phi(A)\right\|^{2} \leq \frac{C_{1}}{N}+\frac{C_{2}}{N^{2}},
$$

which goes to zero as $N \rightarrow \infty$. The hypotheses of Proposition 12 are easily verified.

Let $\psi_{n}(A)$ be the stochastic sequence of binomial type associated with the Poisson measure $\phi$. Then by Theorem 7 ,

$$
\begin{aligned}
\psi_{n}(A) & =\sum_{k=1}^{n}(-1)^{k-1}(n-1)_{k-1} \phi(A) \psi_{n-k}(A) \\
& =\phi(A) \psi_{n-1}(A)+\sum_{j=1}^{n-1}(-1)^{j}(n-1)_{j} \phi(A) \psi_{n-1-j}(A) \\
& =(\phi(A)-n+1) \psi_{n-1}(A) .
\end{aligned}
$$

Thus, we have shown that for the Poisson measure we have

$$
\psi_{n}(A)=(\phi(A))_{n}
$$

EXAMPLE PC (Poisson-Charlier). With the same assumptions as in Example $P$, set

$$
\phi_{C}(A)=\phi(A)-\sigma(A) .
$$

One finds that the stochastic sequence associated with the Poisson-Charlier measure is

$$
\psi_{n}(A)=\mathscr{C}_{n}^{(\alpha)}\left(\phi_{C}(A)\right)
$$

where $\alpha=\sigma(A)$. The preceding equation can be taken as a definition of the Poisson-Charlier polynomials $\mathscr{C}_{n}^{(\alpha)}(x)[5,27,10]$. All properties of PoissonCharlier polynomials can be derived from this definition.

For the Poisson-Charlier measure, we have

$$
\begin{aligned}
& \Delta_{1}(A)=\phi_{C}(A)=\phi(A)-\sigma(A), \\
& \Delta_{n}(A)=\phi(A), \quad n>2,
\end{aligned}
$$

where $\phi$ is the Poisson measure. 
EXAMPLE K (Kravchuk) [26,5]. Let $S$ be a finite set and let $X_{1}, X_{2}, \ldots$ be independent identically distributed Bernoulli random variables such that $\langle X\rangle=p$. Define

$$
\phi(A)=\sum_{i \in A} X_{i}-p|A| .
$$

We find that the stochastic sequence of binomial type associated with the Kravchuk measure is

$$
\psi_{n}(A)=k_{n}^{(p)}(\phi(A)),
$$

where $k_{n}^{(p)}(x)$ are the Kravchuk polynomials.

For the Kravchuk measure, we have

$$
\Delta_{n}(A)=\sum_{i \in A}\left(X_{i}-p\right)^{n}, \quad n \geq 1 .
$$

EXAMPLE CP (Compound Poisson) [11]. Let $\phi$ be a Poisson process as in Example $\mathrm{P}$, and let $X_{1}, X_{2}, \ldots$ be independent identically distributed positive integer-valued random variables. We stress the assumption that $P\left(X_{i}=0\right)=$ 0 . Let $\alpha$ be the common probability distribution of the random variables $X_{i}$, so that $\alpha_{j}=P\left(X_{i}=j\right)$.

The compound Poisson process with distribution $\alpha$ is a measure $\phi_{\alpha}$ defined on $(S, \Sigma)$ as

$$
\phi_{\alpha}(A)=X_{1}+X_{2}+\cdots+X_{\phi(A)}
$$

for every $A \in \Sigma$ such that $\sigma(A)<\infty$.

Here we find that

$$
\mathrm{St}_{\pi}^{[n]}(A \times \cdots \times A)=m_{\pi}\left(X_{1}, X_{2}, \ldots, X_{\phi(A)}\right),
$$

and the stochastic sequence of binomial type associated with the compound Poisson process is

$$
\psi_{n}(A)=n ! e_{n}\left(X_{1}, X_{2}, \ldots, X_{\phi(A)}\right) .
$$

The diagonal measures for the compound Poisson process are

$$
\Delta_{n}(A)=X_{1}^{n}+X_{2}^{n}+\cdots+X_{\phi(A)}^{n} .
$$

EXAMPLE U (U mbral). This example requires some acquaintance with the notation introduced in [33]. Let $\phi$ and $\sigma$ be as in Example P, and let $\alpha_{1}, \alpha_{2}, \ldots$ be exchangeable umbrae. Define

$$
\phi_{\alpha}(A)=\alpha_{1}+\alpha_{2}+\cdots+\alpha_{\phi(A)} .
$$

Again, one finds that

$$
\psi_{n}(A)=n ! e_{n}\left(\alpha_{1}, \ldots, \alpha_{\phi(A)}\right) .
$$

For the umbral process, we have

$$
\Delta_{n}(A)=\alpha_{1}^{n}+\alpha_{2}^{n}+\cdots+\alpha_{\phi(A)}^{n} .
$$


8. Combinatorial orthogonality. A measure $\phi(A)$ defined for $A \in \Sigma$, whose values are random variables, is said to be a completely random measure when the following hold.

1. For every set $A \in \Sigma$ such that $\sigma(A)<\infty, \phi(A)$ is a random variable with finite expectation.

2. If $A_{1}, A_{2}, \ldots, A_{n}$ are pairwise disjoint measurable sets of finite measure, then the random variables $\phi\left(A_{1}\right), \phi\left(A_{2}\right), \ldots, \phi\left(A_{n}\right)$ are independent.

A completely random measure $\phi$ is said to be multiplicative if for every positive integer $n$ and for every partition $\pi$ of $[n]$,

$$
\left\langle\mathrm{St}_{\pi}^{[n]}\right\rangle=\bigotimes_{b \in \pi}\left\langle\mathrm{St}_{\hat{1}}^{b}\right\rangle .
$$

All stochastic measures in the examples of the previous section are multiplicative, except for the measures in Examples D and SP. The following proposition provides a necessary and sufficient condition for $\phi$ to be multiplicative.

Proposition 8. The measure $\phi$ is multiplicative if and only if the realvalued measures $\left\langle\Delta_{n}\right\rangle$ are nonatomic for all positive integers $n$.

Proof. For ease of computation, we assume that $\mathscr{A}$ is a rectangle set: $\mathscr{A}=A_{1} \times \cdots \times A_{n}$. We then have

$$
\begin{aligned}
\left\langle\mathrm{St}_{\pi}^{[n]}\right\rangle(\mathscr{A}) & =\left\langle\mathrm{St}_{\pi}^{[n]}\left(\mathscr{A}_{\pi}\right)\right\rangle \\
& =\left\langle\prod_{b \in \pi} \operatorname{St}_{\hat{1}}^{b}\left(\left({\underset{i \in b}{\mathrm{x}}}_{i} A_{i}\right)_{\hat{1}}\right)\right\rangle \\
& =\left\langle\prod_{b \in \pi} \Delta_{|b|}\left(\bigcap_{i \in b} A_{i}\right)\right\rangle \\
& =\prod_{b \in \pi}\left\langle\Delta_{|b|}\left(\bigcap_{i \in b} A_{i}\right)\right\rangle \\
& =\bigotimes_{b \in \pi}\left\langle\mathrm{St}_{\hat{1}}^{b}\right\rangle\left(\mathscr{A}_{\pi}\right) .
\end{aligned}
$$

But

$$
\bigotimes_{b \in \pi}\left\langle\mathrm{St}_{\hat{1}}^{b}\right\rangle(\mathscr{A})=\bigotimes_{b \in \pi}\left\langle\mathrm{St}_{\hat{1}}^{b}\right\rangle\left(\mathscr{A}_{(\geq \pi)}\right)
$$

We need only show, therefore, that

$$
\bigotimes_{b \in \pi}\left\langle\mathrm{St}_{\hat{1}}^{b}\right\rangle\left(\mathscr{A}_{(>\pi)}\right)=0,
$$

where $\mathscr{A}_{(>\pi)}$ is defined as $\mathscr{A}_{(\geq \pi)} \backslash \mathscr{A}_{\pi}$.

If $\sigma>\pi$, then

$$
\bigotimes_{b \in \pi}\left\langle\mathrm{St}_{\hat{1}}^{b}\right\rangle\left(\mathscr{A}_{\sigma}\right)=\bigotimes_{b \in \pi}\left\langle\Delta_{|b|}\right\rangle\left(\tilde{\mathscr{A}_{\sigma^{\prime}}}\right),
$$


where $\tilde{\mathscr{A}} \in S^{[r]}$ and $\sigma^{\prime}>\hat{0}$ is a partition of $[r]$, where $r=|\pi|$. This is zero if and only if the diagonal measures are nonatomic for all $n$.

Theorem 4 expresses the product of two or more stochastic measures as the sum over stochastic measures. When $\phi$ is multiplicative, the expectation of these stochastic measures can be expressed as the product of expectations of diagonal measures. We state these results first for the general case, and then specialize to results concerning $\psi_{n}(A)$ and $\Delta_{n}(A)$.

Proposition 9. Let $\phi$ be a multiplicative completely random measure Let $\pi$ be a partition of $[n]$. Then

$$
\left\langle\bigotimes_{b \in \pi} \mathrm{St}_{\hat{0}}^{b}\right\rangle=\sum_{\{\sigma: \sigma \wedge \pi=\hat{0}\}} \bigotimes_{b \in \sigma}\left\langle\mathrm{St}_{\hat{1}}^{b}\right\rangle
$$

COROLlaRY 1 (Feynman). If $\langle\phi\rangle=0$, and if $b$ is a subset of [ $n]$, then

$$
\left\langle\mathrm{St}_{\hat{0}}^{b} \otimes \mathrm{St}_{\hat{0}}^{b^{c}}\right\rangle= \begin{cases}\sum_{f \in \mathrm{bi}\left(b, b^{c}\right)} \bigotimes_{i \in b}\left\langle\mathrm{St}_{\hat{1}}^{\{i, f(i)\}}\right\rangle, & \text { if }|b|=\left|b^{c}\right|, \\ 0, & \text { otherwise, }\end{cases}
$$

where bi $\left(b, b^{c}\right)$ is the set of bijections from $b$ to $b^{c}$.

Proof. In the notation of the theorem, $\sigma$ is the partition of $[n]$ consisting of the block $\left\{b, b^{c}\right\}$, and $\pi$ is the zero partition. If $\tau \wedge \sigma=\pi$, then each block of $\tau$ must contain no more than one element of each set. On the other hand, if a partition $\tau$ contains any blocks with only one element, then $\left\langle\mathrm{St}_{\tau}^{[n]}\right\rangle=0$, since $\mathrm{St}_{\tau}^{[n]}$ is multiplicative and $\langle\phi\rangle=0$. Therefore, the only contribution to $\left\langle\mathrm{St}_{\tau}^{[n]}\right\rangle$ is from partitions whose blocks each contain exactly one element of each set. If $|b| \neq\left|b^{c}\right|$, no such partitions exist. If $|b|=\left|b^{c}\right|$, there is one such partition for every bijection between $b$ and $b^{c}$.

Corollary 2. If $|b|>1,\left|b^{c}\right| \geq 1$ and $\langle\phi\rangle=0$, then

$$
\left\langle\mathrm{St}_{\hat{0}}^{b} \otimes \phi_{\hat{1}}^{b^{c}}\right\rangle=0 \text {. }
$$

Proof. Again, $\sigma=\left\{b, b^{c}\right\}$, but $\pi=\left\{\{i: i \in b\}, b^{c}\right\}$. If $|b|>1$, each $\tau$ with $\sigma \wedge \tau=\pi$ contains a block with exactly one element, so $\left\langle\mathrm{St}_{\tau}^{[n]}\right\rangle=0$. If $\sigma \wedge \tau=\pi$, one block of $\tau$ will contain $b^{c}$ and at most one element of $b$, and the other blocks will each consist of one of the remaining elements of $b$. If $|b|>1$, therefore, each $\tau$ will contain at least one block which contains only one element, and therefore $\left\langle\mathrm{St}_{\tau}^{[n]}\right\rangle=0$.

We now restate these results in terms of stochastic sequences of binomial type. 
THEOREM 9. Let $\psi_{n}(A)$ be the stochastic sequence of binomial type associated with a multiplicative completely random measure $\phi$. Let $\pi$ be a partition of the set $[n]$, whose blocks are of size $n_{1}, n_{2}, \ldots, n_{k}$. Then

$$
\left\langle\psi_{n_{1}}(A) \cdots \psi_{n_{k}}(A)\right\rangle=\sum_{\{\sigma: \sigma \wedge \pi=\hat{0}\}} \prod_{b \in \sigma}\left\langle\Delta_{|b|}\right\rangle(A) .
$$

Proposition 10 (Orthogonality). Let $\psi_{n}(A)$ be the stochastic sequence of binomial type associated with a multiplicative completely random measure $\phi$. If $\langle\phi\rangle=0$, then

$$
\left\langle\psi_{n}(A) \psi_{m}(A)\right\rangle= \begin{cases}n !\left\langle\Delta_{2}(A)\right\rangle^{n}, & \text { if } m=n, \\ 0, & \text { if } m \neq n\end{cases}
$$

Proposition 11. Under the same assumptions as Proposition 10, if $n>1$ and $m \geq 1$, then

$$
\left\langle\psi_{n}(A) \Delta_{m}(A)\right\rangle=0
$$

EXAMPLE G (Continued). We illustrateTheorem 9 by computing the expectation of a finite product of Hermite polynomials of arbitrary degrees. Recall that

$$
\psi_{n}(A)=H_{n}^{(\sigma(A))}(\phi(A)) .
$$

It will be convenient to use the stochastic sequence of binomial type notation $\psi_{n}(A)$ instead of the Hermite notation. Our objective is to compute

$$
\left\langle\psi_{n_{1}}(A) \psi_{n_{2}}(A) \cdots \psi_{n_{k}}(A)\right\rangle .
$$

Theorem 9 can be applied in the following way. Choose any partition $\pi$ of the set $\left[n_{1}+n_{2}+\cdots+n_{k}\right]$ having blocks $b_{i}$ of size $n_{i}$. Define $n=n_{1}+n_{2}+\cdots+n_{k}$. Then Theorem 9 states that

$$
\left\langle\psi_{n_{1}}(A) \psi_{n_{2}}(A) \cdots \psi_{n_{k}}(A)\right\rangle=\sum_{\{\sigma: \sigma \wedge \pi=\hat{0}\}} \bigotimes_{b \in \sigma}\left\langle\Delta_{|b|}\right\rangle(A) .
$$

However, $\left\langle\Delta_{|b|}\right\rangle=0$ unless $|b|=2$, in which case $\Delta_{2}=\sigma$. The problem therefore boils down to listing all partitions $\sigma$, all of whose blocks have two elements such that $\sigma \wedge \pi=\hat{0}$. If $C$ is equal to the number of such partitions, then we have that

$$
\left\langle\psi_{n_{1}}(A) \psi_{n_{2}}(A) \cdots \psi_{n_{k}}(A)\right\rangle=C \sigma(A)^{n} .
$$

For example, if $n$ is odd, then $C=0$.

The preceding computation is usually carried out using Feynman diagrams. We hope to have finally pinpointed the source of such computations. 
EXAMPLE P (Continued). We compute

$$
\left\langle\psi_{n}(A) \psi_{k}(A)\right\rangle,
$$

where $\psi_{n}(A)$ is the sequence of binomial type associated with the Poisson measure.

Let $\pi$ be a partition of the set $[n+k]$ having a block $b_{1}$ with $k$ elements and a block $b_{2}$ with $n$ elements. Say $k \leq n$. Then Theorem 9 tells us that

$$
\left\langle\psi_{n}(A) \psi_{k}(A)\right\rangle=\sum_{\sigma \wedge \pi=0} \bigotimes_{b \in \sigma}\left\langle\Delta_{|b|}\right\rangle(A) .
$$

Any partition $\sigma$ of the set $[n+k]$ such that $\sigma \wedge \pi=\hat{0}$ has blocks containing either one element or two elements. Every such partition can be obtained by choosing subsets $c_{1}$ and $c_{2}$ of $b_{1}$ and $b_{2}$ such that $\left|c_{1}\right|=\left|c_{2}\right|$, and matching the elements of $c_{1}$ with the elements of $c_{2}$ in an arbitrary way. In this way we obtain

$$
\left\langle\psi_{n}(A) \psi_{k}(A)\right\rangle=\sum_{i \geq 0}\left(\begin{array}{c}
n \\
i
\end{array}\right)\left(\begin{array}{c}
k \\
i
\end{array}\right) i ! \sigma(A)^{n+k-i},
$$

where we have used the fact that $\Delta_{1}(A)=\Delta_{2}(A)=\phi(A)$ and $\langle\phi(A)\rangle=\sigma(A)$.

9. Diagonal measures. Under reasonable assumptions, the diagonal measures $\Delta_{n}$ of a completely random $L^{2}$ measure are given by

$$
\Delta_{n}(A)=\lim _{N \rightarrow \infty} \sum_{i=1}^{N} \phi\left(A_{i N}\right)^{n},
$$

where the limit is taken in the $L^{2}$ sense, and where, for each $N,\left\{A_{i N}: i=\right.$ $1, \ldots, N\}$ is a disjoint partition of $A$ into sets of equal measure.

Proposition 12. Let $\phi$ be good completely random measure, taking values in $L^{2}(\Omega)$. Let $\sigma$ be a nonatomic measure and let the sets $\left\{A_{i N}: i=1, \ldots, N\right\}$ bedefined as above. Let a positive integer $n$ be given, and suppose that for each positive integer $m<n$ there exist positive constants $c_{m}$ and $d_{m}$ such that

$$
\left\langle\left(\Delta_{m}-\left\langle\Delta_{m}\right\rangle\right)^{2}\right\rangle \leq c_{m} \sigma \text { and }\left|\left\langle\Delta_{m}\right\rangle\right| \leq d_{m} \sigma .
$$

Then

$$
\Delta_{n}(A)=\lim _{N \rightarrow \infty} \sum_{i=1}^{N} \phi\left(A_{i N}\right)^{n}
$$

where the limit is taken in $L^{2}$. 
Proof. We have

$$
\begin{aligned}
\Delta_{n}(A) & =\Delta_{n}\left(\bigcup_{i=1}^{N} A_{i N}\right) \\
& =\sum_{i=1}^{N}\left[\phi\left(A_{i N}\right)^{n}-\mathrm{St}_{<\hat{1}}^{[n]}\left(A_{i N}^{[n]}\right)\right],
\end{aligned}
$$

where

$$
\mathrm{St}_{<\hat{1}}^{[n]}\left(A_{i N}^{[n]}\right)=\sum_{\hat{0} \leq \pi<\hat{1}} \mathrm{St}_{\pi}^{[n]}\left(A_{i N}^{[n]}\right)
$$

Therefore,

$$
\begin{aligned}
\left\|\Delta_{n}(A)-\sum_{i=1}^{N} \phi\left(A_{i N}\right)^{n}\right\|^{2} & =\left\|\sum_{i=1}^{N} \mathrm{St}_{<\hat{1}}^{[n]}\left(A_{i N}^{[n]}\right)\right\|^{2} \\
& =\sum_{i=1}^{N}\left\|\mathrm{St}_{<\hat{1}}^{[n]}\left(A_{i N}^{[n]}\right)\right\|^{2}+\sum_{\substack{i, j=1 \\
i \neq j}}^{N}\left\langle\mathrm{St}_{<\hat{1}}^{[n]}\left(A_{i N}^{[n]}\right) \mathrm{St}_{<\hat{1}}^{[n]}\left(A_{j N}^{[n]}\right)\right\rangle .
\end{aligned}
$$

We will show that the right-hand side goes to zero as $N \rightarrow \infty$.

We first show that if $\sigma(A)<1$, then

$$
\left\|\mathrm{St}_{\pi}^{[n]}\left(A^{[n]}\right)\right\|^{2} \leq K_{n} \sigma(A)^{|\pi|},
$$

where $K_{n}$ is a constant depending only on $n$. To this end, set $\tilde{\Delta}_{m}=\Delta_{m}-\left\langle\Delta_{m}\right\rangle$. Suppose $|\pi|=r$, and say that the blocks of $\pi$ have sizes $m_{1}, \ldots, m_{r}$. Then

$$
\begin{aligned}
\mathrm{St}_{\pi}^{[n]}\left(A^{[n]}\right) & =\left(\Delta_{m_{1}} \otimes \cdots \otimes \Delta_{m_{r}}\right)\left(A_{\hat{0}}^{[r]}\right) \\
& =\sum_{b \subset[r]} \Phi_{b}^{[r]}\left(A_{\hat{0}}^{[r]}\right),
\end{aligned}
$$

where

$$
\Phi_{b}^{[r]}=\bigotimes_{j \in b}\left\langle\Delta_{m_{j}}\right\rangle \otimes \bigotimes_{j \in b^{c}} \tilde{\Delta}_{m_{j}} \equiv \bar{\Psi}^{b} \otimes \tilde{\Psi}^{b^{c}} .
$$

Let $C_{n}=\max _{m<n}\left\{1, c_{m}, d_{m}\right\}$. Then

$$
\left\|\bar{\Psi}^{b}\left(A^{b}\right)\right\| \leq C_{n}^{|b|} \sigma(A)^{|b|} .
$$

We will show that

$$
\left\|\tilde{\Psi}^{b}\left(A_{\hat{0}}^{b}\right)\right\| \leq \sqrt{|b| ! C_{n}^{|b|} \sigma(A)^{|b|}} .
$$

Recalling that $C_{n} \geq 1$ and $\sigma(A)<1$, this inequality implies that

$$
\left\|\Phi_{b}^{[r]}\left(A_{\hat{0}}^{[r]}\right)\right\| \leq C_{n}^{r} \sqrt{r !} \sigma(A)^{r / 2}
$$


and thus that

$$
\left\|\mathrm{St}_{\pi}^{[n]}\left(A^{[n]}\right)\right\| \leq 2^{|r|} \sqrt{r !} C_{n}^{r} \sigma(A)^{r / 2} .
$$

The assertion follows by setting $K_{n}=2^{n} \sqrt{n !} C_{n}^{n}$.

To prove $(* *)$, write

$$
A_{\hat{0}}^{[s]}=\bigcup\left(B_{j_{1}} \times \cdots \times B_{j_{s}}\right),
$$

where $\left\{B_{j}\right\}$ is a countable set of measurable disjoint sets whose union is $A$, and the union on the right-hand side ranges over all sets of indices such that $j_{1}, \ldots, j_{s}$ are all distinct. Then

$$
\begin{aligned}
\left\|\tilde{\Psi}^{[s]}\left(A_{\hat{0}}^{[s]}\right)\right\|^{2} & =\sum_{j_{1}, \ldots, j_{s}} \sum_{k_{1}, \ldots, k_{s}}\left\langle\tilde{\Delta}_{m_{1}}\left(B_{j_{1}}\right) \cdots \tilde{\Delta}_{m_{s}}\left(B_{j_{s}}\right) \tilde{\Delta}_{m_{1}}\left(B_{k_{1}}\right) \cdots \tilde{\Delta}_{m_{s}}\left(B_{k_{s}}\right)\right\rangle \\
& =s ! \sum_{j_{1}, \ldots, j_{s}}\left\langle\tilde{\Delta}_{m_{1}}^{2}\left(B_{j_{1}}\right)\right\rangle \cdots\left\langle\tilde{\Delta}_{m_{s}}^{2}\left(B_{j_{s}}\right)\right\rangle \\
& \leq s ! C_{n}^{s} \sum_{j_{1}, \ldots, j_{s}} \sigma\left(B_{j_{1}}\right) \cdots \sigma\left(B_{j_{s}}\right) \\
& \leq s ! C_{n}^{s} \sigma(A)^{s} .
\end{aligned}
$$

Returning to $(*)$, we note that

$$
\left\|x_{1}+\cdots+x_{n}\right\|^{2} \leq n\left(\left\|x_{1}\right\|^{2}+\cdots+\left\|x_{n}\right\|^{2}\right) .
$$

Thus,

$$
\begin{aligned}
\sum_{i=1}^{N}\left\|\mathrm{St}_{<\hat{1}}^{[n]}\left(A_{i N}^{[n]}\right)\right\|^{2} & \leq N\left(B_{n}-1\right) \sum_{\hat{0} \leq \pi<\hat{1}}\left\|\mathrm{St}_{\pi}^{[n]}\left(A_{i N}^{[n]}\right)\right\|^{2}, \\
& \leq N\left(B_{n}-1\right)^{2} K_{n} \sigma\left(A_{i N}\right)^{2} \\
& =\frac{1}{N}\left(B_{n}-1\right)^{2} K_{n} \sigma(A)^{2},
\end{aligned}
$$

where $B_{n}$, Bell's number, is the number of partitions of the set [ $n$ ] (see [29]).

We now bound the cross terms. If $A$ and $A^{\prime}$ are disjoint, then

$$
\left|\left\langle\mathrm{St}_{\pi}^{[n]}\left(A^{[n]}\right) \mathrm{St}_{\pi^{\prime}}^{[n]}\left(A^{\prime[n]}\right)\right\rangle\right| \leq C_{n}^{r+s} \sigma(A)^{|\pi|} \sigma\left(A^{\prime}\right)^{\left|\pi^{\prime}\right|},
$$

where $r=|\pi|$ and $s=|\sigma|$. Decomposing both $A^{[n]}$ and $A^{\prime[n]}$ in terms of triangular sets, as above, we get

$$
\begin{aligned}
& \left\langle\mathrm{St}_{\pi}^{[n]}\left(A^{[n]}\right) \mathrm{St}_{\pi^{\prime}}^{[n]}\left(A^{\prime[n]}\right)\right\rangle \\
& \quad=\sum_{j_{1}, \ldots, j_{r}} \sum_{k_{1}, \ldots, k_{s}}\left\langle\Delta_{m_{1}}\left(B_{j_{1}}\right) \cdots \Delta_{m_{r}}\left(B_{j_{r}}\right) \Delta_{m_{1}^{\prime}}\left(B_{k_{1}}^{\prime}\right) \cdots \Delta_{m_{s}^{\prime}}\left(B_{k_{s}}^{\prime}\right)\right\rangle .
\end{aligned}
$$


Since the $r+s$ sets of each term are pairwise disjoint, we obtain

$$
\begin{aligned}
& \left|\left\langle\mathrm{St}_{\pi}^{[n]}\left(A^{[n]}\right) \mathrm{St}_{\pi^{\prime}}^{[n]}\left(A^{\prime[n]}\right)\right\rangle\right| \\
& \quad \leq C_{n}^{r+s} \sum_{j_{1}, \ldots, j_{r}} \sum_{k_{1}, \ldots, k_{s}} \sigma\left(B_{j_{1}}\right) \cdots \sigma\left(B_{j_{r}}\right) \sigma\left(B_{k_{1}}^{\prime}\right) \cdots \sigma\left(B_{k_{s}}^{\prime}\right) \\
& \quad \leq C_{n}^{2 n} \sigma(A)^{|\pi|} \sigma\left(A^{\prime}\right)^{\left|\pi^{\prime}\right|} .
\end{aligned}
$$

Thus,

$$
\left|\left\langle\mathrm{St}_{\pi}^{[n]}\left(A_{i N}^{[n]}\right) \mathrm{St}_{\pi^{\prime}}^{[n]}\left(A_{j N}^{[n]}\right)\right\rangle\right| \leq \frac{1}{N^{4}} C_{n}^{2 n} \sigma(A)^{4},
$$

and

$$
\sum_{\substack{i, j=1 \\ i \neq j}}^{N}\left|\left\langle\mathrm{St}_{<\hat{1}}^{[n]}\left(A_{i N}^{[n]}\right) \mathrm{St}_{<\hat{1}}^{[n]}\left(A_{j N}^{[n]}\right)\right\rangle\right| \leq \frac{\left(B_{n}-1\right)^{2}}{N^{2}} C_{n}^{2 n} \sigma(A)^{4} .
$$

\section{REFERENCES}

[1] BJorken, J. D. and Drell, S. D. (1964). Relativistic Quantum Mechanics. McGraw-Hill, New York.

[2] Bogoliubov, N. N. and Shirkov, D. V. (1980). Introduction to the Theory of Quantized Fields. Wiley, New York.

[3] Cameron, R. H. and Martin, W. T. (1947). The orthogonal development of nonlinear functionals in series of F ourier-Hermite functionals. Ann. Math. 48 385-392.

[4] CARlen, E. A. and KrÉE, P. (1991). $L^{p}$ estimates on iterated stochastic integrals. Ann. Probab. 19 354-368.

[5] Chinara, T. S. (1978). An Introduction to Orthogonal Polynomials. Gordon and Breach, New York.

[6] Cox, D. R. and Isham, V. (1980). Point Processes. Chapman and Hall, London.

[7] DolÉANs-DADE, C. (1970). Quelques applications de la formule de changement de variables pour les semimartingales. Z. Wahrsch. Verw. Gebiete 16 181-194.

[8] Doubilet, P. (1972). On the foundations of combinatorial theory. VII: symmetric functions through the theory of distribution and occupancy. Stud. Appl. Math. 51 377-396.

[9] Engel, D. D. (1982). The multiple stochastic integral. Mem. Am. Math. Soc. 38 1-82.

[10] Floreanini, R., LeTourneux, J. and Vinet, L. (1993). Quantum mechanics and polynomials of a discrete variable. Ann. Physics 226 331-349.

[11] HidA, T. (1970). Stationary Stochastic Processes. Princeton Univ. Press.

[12] HidA, T. and IKEDA, N. (1965). Analysis on Hilbert space with reproducing kernel arising from multiple Wiener integral. Proc. Fifth Berkeley Symp. Math. Statist. Probab. 2 117-143. Univ. California Press, Berkeley.

[13] Iтô, K. (1951). Multiple Wiener integral. J . Math. Soc. J apan 3 157-169.

[14] ITô, K. (1956). Spectral type of the shift transformation of differential processes with stationary increments. Trans. Amer. Math. Soc. 81 253-263.

[15] KaKutani, S. (1950). Determination of the spectrum of the flow of Brownian motion. Proc. Nat. Acad. Sci. U.S.A. 36 319-323.

[16] KaKUTANI, S. (1961). Spectral analysis of stationary Gaussian processes. Proc. Fourth Berke ley Symp. Math. Statist. Probab. 2 239-247. Univ. California Press, Berkeley.

[17] Kallenberg, O. (1983). Random Measures. Academic Press, New York.

[18] Kallenberg, O. and Szulga, J. (1989). Multiple integration with respect to Poisson and Lévy processes. Probab. Theory Related Fields 83 101-134.

[19] Lebedev, N. N. (1972). Special Functions and Their Applications. Dover, New York. 
[20] MAJOR, P. (1981). Multiple Wiener-Itô I ntegrals. Lecture Notes in Math. 849. Springer, New York.

[21] McKean, JR., H. P. (1955). Sample functions of stable processes. Ann. Math. 61 564-579.

[22] McKean, JR., H. P. (1969). Stochastic Integrals. Academic Press, New York.

[23] Metivier, M. and Pellaumail, J. (1980). Stochastic I ntegration. Academic Press, New York.

[24] Meyer, P.-A. (1976). Un cours sur les integrales stochastiques. Seminaire de Probabilités X: Université de Strasbourg. Lecture Notes in Math. 511 245-400. Springer, Berlin.

[25] Meyer, P.-A. (1993). Quantum Probability for Probabilists. Lecture Notes in Math. 1538. Springer, New York.

[26] Nikiforov, A. F., Suslov, S. K. and Uvarov, V. B. (1991). Classical Orthogonal Polynomials of a Discrete Variable Springer, Berlin.

[27] OGuRA, H. (1972). Orthogonal functionals of the Poisson process. IEEE Trans. Inform. The ory IT-18 473-481.

[28] Protter, P. (1990). Stochastic Integration and Differential Equations: A New Approach. Springer, Berlin.

[29] Rota, G.-C. (1964). The number of partitions of a set. Amer. Math. Monthly 71 498-504.

[30] RotA, G.-C. (1964). On the foundations of combinatorial theory I. Theory of Möbius functions. Z. Wahrsch. Verw. Gebiete 2 340-368.

[31] Rota, G.-C. (1975). Finite Operator Calculus. Academic Press, New York.

[32] Rota, G.-C., KAhANer, D. and Odlyzko, A. (1973). On the foundations of combinatorial theory. VIII. Finite operator calculus. J. Math. Anal. Appl. 42 684-760.

[33] RotA, G.-C. and TAYLOR, B. D. (1994). The classical umbral calculus. SIAM J . Math. Anal. 25 694-711.

[34] SEgAL, I. E. (1956). Tensor algebras over Hilbert spaces, I. Trans. Amer. Math. Soc. 81 106134

[35] Segall, A. and KaIlath, T. (1976). Orthogonal functionals of independent increment processes. IEEE Trans. Inform. Theory IT-22 287-298.

[36] Streater, R. F. and Wightman, A. S. (1964). PCT, Spin and Statistics, and All That. Benjamin/Cummings, Reading, MA.

[37] Wick, G.-C. (1950). Phys. Rev. 80 268-272.

[38] WiEner, N. (1938). The homogeneous chaos. Amer. J . Math. 55 897-936.

[39] Wiener, N. and Wintner, A. (1941). The discrete chaos. Amer. J. Math. 65 279-298.

[40] Zinn-Justin, J. (1989). Quantum Field Theory and Critical Phenomena. Clarendon Press, Oxford.

DEPARTMENT OF MATHEMATiCS

MASSACHUSETTS INSTITUTE

OF TECHNOLOGY

CAmbridge, Massachusetts 02139

E-MAIL: rota@math.mit.edu
THEORETICAL Division

Los Alamos National Laboratory

Los Alamos, New Mexico 87545

E-MAIL: tcw@anl.gov 